Nephrologe 2009 • 4:299-300

DOI 10.1007/s11560-009-0310-z

Online publiziert: 15. Mai 2009

(c) Springer Medizin Verlag 2009

\author{
G. Wolf ${ }^{1} \cdot$ E. Schleußner ${ }^{2}$ \\ ${ }^{1}$ Klinik für Innere Medizin IIII, Universitätsklinikum Jena \\ ${ }^{2}$ Klinik für Frauenheilkunde und Geburtshilfe, Abteilung \\ Geburtshilfe, Universitätsklinikum Jena
}

\title{
Schwangerschaft und Niere
}

Das vorliegende Heft „Der Nephrologe“ ist dem Thema "Schwangerschaft und Niere" gewidmet. Es bestehen vielfältige Wechselwirkungen zwischen Schwangerschaft und Nierenfunktion. Zum einen können durch die Schwangerschaft vorbestehende Nierenerkrankungen wie verschiedene Arten der Glomerulonephritis verschlimmert werden und es können typische schwangerschaftsspezifische Erkrankungen, die die Nieren betreffen, wie zum Beispiel eine Präeeklampsie, sich erstmalig manifestieren. Zum anderen zeigen aber auch epidemiologische Untersuchungen, dass ein klarer Zusammenhang zwischen niedrigem Geburtsgewicht mit gestörter Nierenentwicklung und dem Auftreten von chronischen Nierenerkrankungen im Erwachsenenalter besteht. Im ersten Fall trägt die mütterliche Niere die Konsequenzen der pathophysiologisch verlaufenden Schwangerschaft, während im zweiten Fall die spätere Nierenfunktion des sich entwickelnden Kindes beeinträchtigt sein kann. Im vorliegenden Heft werden verschiedene Aspekte der komplexen Wechselwirkung zwischen Schwangerschaft und Niere dargestellt.

Die physiologische Umstellung nahezu aller Organsysteme während der Schwangerschaft dient der Befriedigung der fetalen Ansprüche im Bezug auf Wachstum und Entwicklung. Gerth et al. beschreiben in ihrem Beitrag diese Adaptationsvorgänge, deren Verständnis wichtig ist, insbesondere auch im Hinblick auf die diagnostische Abgrenzung pathophysiologischer, teilweise diskreter Verände- rungen bei Nierenerkrankungen, die in der Schwangerschaft auftreten.

\section{(7) Wichtig ist die Abgrenzung physiologischer Umstellungen von - teils diskreten - pathologischen Veränderungen}

Im Beitrag von Dötsch et al. wird der aktuelle Stand zum Thema „Fetale Programmierung und spätere Nierenfunktionsstörung nach intrauteriner Wachstumsrestriktion" dargestellt. In den letzten Jahren konnte gezeigt werden, dass die fetale Programmierung ein wichtiger Faktor in der Genese von Bluthochdruck und chronischen Nierenerkrankungen im späteren Erwachsenenleben ist.

Hypertensive Erkrankungen wie Präeklampsie gehören $\mathrm{zu}$ den häufigsten Schwangerschaftskomplikationen. Der aktuelle Stand der Pathophysiologie und Therapie dieser relativ häufigen Komplikationen wird von Schleußner ausführlich dargestellt.

Glomerulonephritiden können während der Schwangerschaft erstmalig auftreten bzw. es können sich vorbestehende Glomerulonephritiden während der Schwangerschaft verschlechtern. Panzer et al. beschreiben in ihrer Arbeit die wichtigsten Aspekte hinsichtlich Diagnostik und die schwierige Entscheidung bezüglich möglicher Therapieoptionen. Ein besonderer Schwerpunkt liegt dabei auf Schwangerschaften von Patientinnen mit einem systemischen Lupus erythematodes.

Schwangerschaften von Dialysepatientinnen sind sehr selten, da es durch die 
terminale Niereninsuffizienz zur hormonellen Veränderung kommt, die eine Infertilität begünstigen. Im Gegensatz hierzu sind Schwangerschaften nach Nierentransplantation häufiger beschrieben. Das wichtige Thema Schwangerschaft bei Nierenersatztherapie/Nierentransplantation wird von Frau Ott umfassend dargestellt. In einer Schwangerschaft können Erkrankungen des ableitenden Harntraktes eine besondere Rolle spielen. Die Schwangerschaft beispielsweise prädisponiert zur Entwicklung komplizierter Harnwegsinfekte. Im Beitrag von Steiner et al. wird ein Überblick über urologische Erkrankungen in der Schwangerschaft gegeben.

\section{(7) Schwangere mit}

\section{Nierenerkrankungen sollten} interdisziplinär in tertiären Zentren betreut werden

Wir hoffen, dass der interessierte Leser im vorliegenden Schwerpunktheft ei- nen Überblick über das komplexe Thema „Niere und Schwangerschaft" erhält. Aufgrund der Komplexizität der Erkrankung sollte eine Betreuung von Schwangeren mit Nierenerkrankungen immer interdisziplinär durch Geburtshelfer, Nephrologen und Urologen in tertiären Zentren mit Erfahrungen auf diesem Gebiet erfolgen. Konsequenterweise wird dieses Heft in enger Zusammenarbeit von einem Nephrologen mit einem Geburtshelfer herausgegeben.

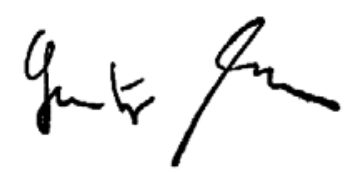

G. Wolf

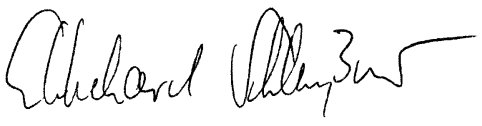

E. Schleußner

\section{Korrespondenzadressen}

Prof. Dr. G. Wolf

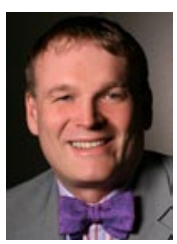

Klinik für Innere Medizin IIII, Universitätsklinikum Jena 07740 Jena

Gunter.Wolf@med.uni-jena.de

Prof. Dr. E. Schleußner

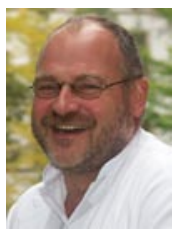

Klinik für Frauenheilkunde und Geburtshilfe, Abteilung Geburtshilfe,

Universitätsklinikum Jena 07740 Jena

Ekkehard.Schleussner@ med.uni-jena.de

\section{(>) Exklusiv für Abonnenten :}

\section{Nutzen Sie das Online-Archiv von Der Nephrologe}

Ihre Vorteile:

- Komfortable und schnelle Recherche nach Themen, Autoren, Suchbegriffen

— Ob unterwegs oder am eigenen PC: Zugriff überall und jederzeit

— Online First: Lesen Sie die aktuellsten Beiträge schon vor Erscheinen des gedruckten Heftes online

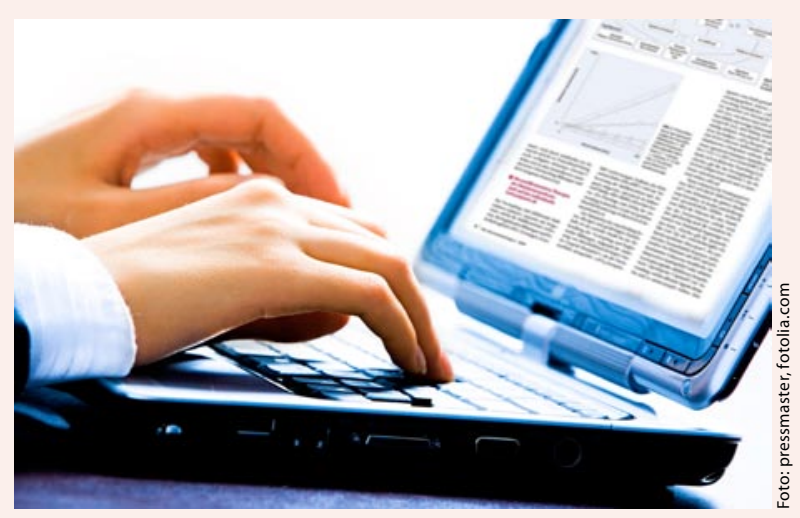

